\title{
Treatment Satisfaction, Patient Preferences, and the Impact of Suboptimal Disease Control in a Large International Rheumatoid Arthritis Cohort: SENSE Study
}

This article was published in the following Dove Press journal:

Patient Preference and Adherence

Peter C Taylor, (D)

Codrina Ancuta, ${ }^{2}$ Orsolya Nagy, ${ }^{3}$

María C de la Vega, ${ }^{4}$

Andrey Gordeev, ${ }^{5}$ Radka Janková, ${ }^{6}$ Umut Kalyoncu, ${ }^{7}$ Ivan Lagunes-

Galindo, ${ }^{3}$ Jadranka Morović-

Vergles, ${ }^{8}$ Mariana

Peixoto GU e Silva de Souza, ${ }^{9}$

Bernadette Rojkovich, ${ }^{10}$

Prodromos Sidiropoulos, "

Atsushi Kawakami $^{12}$

'Botnar Research Centre, Nuffield Department of Orthopaedics, Rheumatology and

Musculoskeletal Sciences, University of Oxford,

Oxford, UK; ${ }^{2}$ Department of Rheumatology,

"Grigore T. Popa" University of Medicine and

Pharmacy, lași, Romania; ${ }^{3}$ AbbVie Inc, North

Chicago, IL, USA; ${ }^{4}$ CEIM Investigaciones

Medicas, Buenos Aires, Argentina; ${ }^{5}$ V.A.

Nasonova Research Institute of Rheumatology,

Moscow, Russian Federation; ${ }^{6}$ Department of

Pediatric and Adult Rheumatology, Faculty

Hospital Motol, Prague, Czech Republic;

${ }^{7}$ Department of Internal Medicine, Division of

Rheumatology, Hacettepe University, Ankara,

Turkey; ${ }^{8}$ Department of Internal Medicine,

Division of Clinical Immunology, Allergology

and Rheumatology, Dubrava University

Hospital, University of Zagreb School of

Medicine, Zagreb, Croatia; 'Santa Casa de Belo

Horizonte, Belo Horizonte, Brazil;

${ }^{10}$ Department of Rheumatology and

Physiotherapy, Polyclinic of the Hospitaller

Brothers of St. John of God, Semmelweis

University, Budapest, Hungary; "'Faculty of

Medicine, University of Crete, Heraklion,

Greece; ${ }^{2}$ Department of Immunology and

Rheumatology, Nagasaki University Graduate

School of Biomedical Sciences, Nagasaki, Japan

Correspondence: Peter C Taylor

Botnar Research Centre, Nuffield Department of Orthopaedics, Rheumatology and Musculoskeletal Sciences, University of Oxford, Windmill Road, Headington, Oxford, OX3 7LD, UK

Tel +44I865227323

Email peter.taylor@kennedy.ox.ac.uk
Background: Patients' needs and perspectives are important determinants of treatment success in rheumatoid arthritis (RA). Assessing patients' perspectives can help identify unmet needs and enhance the understanding of treatment benefits.

Objective: The SENSE study assessed the impact of inadequate response to diseasemodifying antirheumatic drugs (DMARDs) on treatment satisfaction, disease outcomes, and patient perspectives related to RA disease management.

Methods: SENSE was a noninterventional, cross-sectional study conducted in 18 countries across Europe, Asia, and South America. Adult patients with poorly controlled RA of moderate/high disease activity were eligible. Patient satisfaction was assessed by the Treatment Satisfaction Questionnaire for Medication (TSQM v1.4). Treatment adherence, healthcare resource utilization (HRU), quality of life (QoL), work ability, digital health literacy (DHL), patient preference information, and treatment strategy were also assessed.

Results: A total of 1624 patients were included in the study: most were female $(84.2 \%)$ and middle-aged, and mean disease duration was 10.5 years. Mean TSQM global satisfaction subscore was 60.9 , with only $13.5 \%$ of patients reporting good treatment satisfaction (TSQM global $\geq 80$ ). The strongest predictor of good treatment satisfaction was treatment with advanced therapies. Most patients $(87.4 \%)$ reported good treatment adherence. In general, patients had impaired QoL and work ability, high HRU, and $67.4 \%$ had poor DHL. Leading treatment expectations were "general improvement of arthritis" and "less joint pain". Most patients preferred oral RA medications $(60.7 \%)$ and rapid ( $\leq 1$ week) onset of action $(71.1 \%)$. "Increased risk for malignancies" and "increased risk for cardiovascular disease" were the least acceptable side effects. Despite suboptimal control, advanced therapies were only used in a minority of patients, and DMARD switches were planned for only half of the patients. Conclusion: Suboptimal disease control negatively impacts treatment satisfaction, work ability, QoL, and HRU. Data collected on patient perspectives may inform shared decisionmaking and optimize treat-to-target strategies for improving patient outcomes in RA.

Keywords: adherence, digital health literacy, patient preference, rheumatoid arthritis, treatment satisfaction

\section{Introduction}

Rheumatoid arthritis (RA) is a treatable condition, largely due to the expansion of the number of available, effective disease-modifying antirheumatic drugs (DMARDs). However, in the real world, rates of remission or low disease activity 
range from $32 \%$ to $55 \%$ at 12 months, suggesting that approximately half of patients with RA may have high unmet needs for more effective disease management, ${ }^{1}$ at least in part because treat-to-target (T2T) principles are not universally applied. Barriers to implementing T2T principles are numerous, including treating physicians' clinical inertia, patients' concerns related to treatment intensification, and reimbursement of effective medications. ${ }^{2}$

As well as effective therapies, patients' characteristics and perspectives, including treatment preferences and expectations, are important determinants of treatment success in RA. ${ }^{3}$ Patient-reported outcomes (PROs) and patient preference information (PPI) are of growing importance and can provide meaningful insights into patients' perspectives, reflecting an improved patient-physician partnership in RA care. PROs are measurements of patients' health conditions as reported directly by patients, without amendment or interpretation by clinicians. PPI refers to qualitative or quantitative assessments of patients' relative preference or acceptability of specified choices among treatment options (eg, risks and benefits) or other attributes (eg, route of drug administration and need for combination with other drugs). ${ }^{4-6}$

Adherence to RA therapy is a key component in the effective management of RA. ${ }^{7}$ Patients' satisfaction with therapy has an impact on medication adherence, treatment continuation, and future treatment choices, but these aspects of satisfaction have, as yet, been studied only in small populations of patients with RA. ${ }^{8,9}$ Satisfaction is also closely linked to patients' treatment expectations, ${ }^{10}$ which relate to patients' everyday lives, personal concerns, and levels of pain. However, patients' expectations can be very different from rheumatologists' treatment goals. ${ }^{11}$

Adherence may be improved via patient support programs (PSPs) ${ }^{12}$ and health education, including easily accessible digital information, which may contribute to patient empowerment and more effective self-care. ${ }^{7}$ The effectiveness of health education via digital information relies on digital health literacy (DHL), ie, on patients' abilities to access and use credible online health information, so they may become better informed to make decisions about their treatment, which, in turn, may positively influence their health behavior. ${ }^{13}$

Evaluating PPI and PROs and understanding what drives patient satisfaction with therapy can provide valuable insight into the impact of RA on patients' lives and a more comprehensive assessment of unmet needs and treatment benefits. ${ }^{3,14-16}$ The SENSE study was designed to better understand the impact of inadequate response to
DMARDs on treatment satisfaction and various disease outcomes and to analyze patient attitudes and perspectives toward treatment and their disease. SENSE also provided an opportunity to assess DHL in a large multinational cohort of patients with RA using a validated tool, the eHealth Literacy Scale (eHEALS).

\section{Methods \\ Design}

SENSE was a noninterventional, cross-sectional study conducted in 18 countries across Europe, Asia, and South America, including Argentina, Brazil, Bulgaria, Chile, Croatia, Czech Republic, Estonia, Greece, Hungary, Japan, Latvia, Lithuania, Poland, Romania, Russian Federation, Slovakia, Turkey, and Uruguay.

\section{Patients}

Eligible patients were adults ( $\geq 18$ years old) diagnosed with RA and fulfilling the 1987-revised American College of Rheumatology (ACR) or the 2010 ACR/ European League Against Rheumatism (EULAR) classification criteria for RA, who attended a routine visit (previously scheduled as a follow-up of their RA disease), and were receiving treatment with an approved conventional synthetic DMARD (csDMARD), targeted synthetic DMARD (tsDMARD), or biologic DMARD (bDMARD). At the time of enrollment, patients had been exposed to $\leq 2$ bDMARDs. Eligible patients had suboptimal disease control, defined as moderate or high disease activity (Disease Activity Score in 28 joints [DAS28] $>3.2$ for $\geq 1$ month but for $\leq 4$ months prior to enrollment) despite receiving a full tolerable dose of DMARD therapy that had been administered for $\geq 3$ months.

Patients had to understand the language of their country of residence, be willing and able to complete PRO questionnaires, and provide written authorization to the investigator to use/disclose personal and/or health data, or to provide informed consent if required by local regulations. Community- or hospital-based medical centers experienced in the treatment of RA were selected as research sites. Convenience sampling was employed; all consecutive patients attending a routine visit and fulfilling enrollment criteria could be included.

Patients were enrolled from September 14, 2018 to May 31, 2019. Data available in the medical records of each patient were collated and documented in an electronic case-report form. For each patient, the study consisted of 
one visit in which the patient was asked to complete PRO and PPI questionnaires. According to the requirements for noninterventional or observational studies, no additional diagnostic or monitoring procedures were applied to the patients included in the study other than those that would ordinarily be applied in the course of the therapeutic strategy.

The study was conducted in accordance with the Declaration of Helsinki and has been approved by local ethics committees according to local laws and regulations for participating countries (Supplementary Table 1). All patients provided written authorization to the investigator to use/disclose personal and/or health data or to provide informed consent if required by local regulations.

\section{Assessments}

Clinical parameters, including comorbidities at the Medical Dictionary for Regulatory Activities system organ class level, and sociodemographic characteristics were collected for all patients, along with details of their current treatment and planned treatment strategy. Physicians were asked to report if switching to a different DMARD was planned for their patient, and the mode of action of planned treatment switches was captured.

The primary outcome was patients' RA treatment satisfaction, which was assessed using the Treatment Satisfaction Questionnaire for Medication (TSQM; v1.4). ${ }^{15}$ The TSQM provides a validated score for four subscales: effectiveness, side effects, convenience, and global satisfaction. Good treatment satisfaction was defined as a global satisfaction score $\geq 80$ out of a possible $100 .{ }^{15}$

Health-related QoL (HRQoL) was assessed using validated PRO measures: the Functional Assessment of

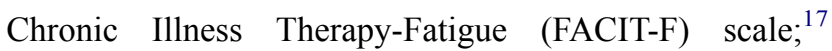
Health Assessment Questionnaire-Disability Index (HAQDI) ${ }^{18}$ 36-item Short Form Health Survey (SF-36; Version 2) Physical and Mental Component Summaries (PCS, MCS); ${ }^{19}$ and the Work Productivity and Activity Impairment (WPAI)-RA (Version 2.0) questionnaire. $^{20}$ Visual analog scales (VAS) using numeric rating scales (NRS) were used to assess severity of morning stiffness and pain in the past 7 days (range $0=$ "no stiffness/pain" to $10=$ "worst possible stiffness/pain"). Duration of morning stiffness in the past 7 days (in minutes) was also recorded. Patients also reported their adherence to medication, which was assessed using a VAS, a validated measure that highly correlated with electronic monitoring results in patients with chronic conditions, including RA. ${ }^{21,22}$ Good adherence was defined as $\geq 80 \%$ on the VAS. ${ }^{23}$

DHL was determined using the eHEALS, ${ }^{13}$ a selfreport tool of 10 questions based on individuals' perceptions of their own skills and knowledge within each measured domain, providing scores ranging from 8 to 40 . The instrument is designed to provide a general estimate of consumer eHealth-related skills and offers insight into the ability of patients to seek, find, understand, use, and appraise health information from electronic sources and to apply the knowledge gained to addressing or solving health problems. ${ }^{13} \mathrm{~A}$ higher total eHEALS indicates greater perceived skills at using online health information to help solve health problems; a score of $<26$ was considered to represent poor DHL. ${ }^{24}$

Patient perspectives were assessed using PPI questionnaires developed by AbbVie (Supplementary Text 1), based on the available literature ${ }^{3,16,25}$ and patient research due to limited availability of appropriate validated tools. Preferences for pharmacologic treatment were assessed via a 6-item direct preference assessment questionnaire related to different treatment attributes. Respondents were asked to select only one alternative at a time, without providing information on the order of the unselected alternatives. The frequency (\%) of answer options given by study patients for each question - which included preferences for route of administration, combination therapy, time to effect, and acceptable side effects - were recorded. These attributes were not combined in a single scenario as a discrete choice experiment. ${ }^{26}$ Expectations for pharmacologic treatment were assessed using an 11-item questionnaire with a 7-point NRS $(1=$ "no improvement needed" to $7=$ "the most improvement needed"). Need for a PSP was assessed using a 17-item questionnaire with a 7-point NRS $(1=$ "not needed at all" to $7=$ "very much needed").

\section{Statistical Analysis}

Approximately 1500 patients with RA were planned to be included in the study, with the sample size calculation based on information of global satisfaction measured by TSQM. Based on a study conducted in patients treated with tumor necrosis factor inhibitor (TNFi) for 3 months, it seemed reasonable to assume that the standard deviation (SD) for the global satisfaction was about 20 , with mean values ranging from 79 to $83 .{ }^{27}$ 
Descriptive statistics were used to describe treatment satisfaction, expectations, and patient preferences using the full analysis set (FAS), which included all patients who fulfilled all inclusion criteria. Patient data and all PROs were assessed by subgroups, which were stratified by treatment, disease activity, and presence of comorbidities. For subgroup analyses of continuous variables, Wilcoxon-Mann-Whitney tests were used; and for categorical variables, Chi-square tests or Fisher exact tests were used. Analysis of variance tests were conducted for each individual score of a questionnaire to assess treatment expectations (dependent variable) and with the grouping variable csDMARD vs bDMARD vs tsDMARD. Spearman's rank correlation coefficient $(\rho)$ was calculated for selected associations (self-reported adherence, DAS28, need for PSP in general, and individual scores of the questionnaire to assess treatment expectations) with various parameters. Multiple logistic regression analyses were conducted to determine variables that might predict good treatment satisfaction, patients' preference for oral therapy, preference for monotherapy, good adherence, and willingness of the treating physician to switch medication. Prespecified prognostic factors were used in the regression models with dichotomized or grouped categorical independent variables (Supplementary Text 2; Supplementary Table 2). Backward elimination with a significance level of 0.05 was applied to stepwise automatically select relevant predictors. Effect estimates and $P$-values using the Chi-square test are provided for each prespecified predictor.

\section{Results}

A total of 1629 patients were enrolled in this study, of which 1624 were included in the FAS.

\section{Clinical Parameters and}

\section{Sociodemographic Characteristics}

Most patients were female (84.2\%) with a mean age of 58.4 years (range 18-90) and a mean (SD) disease duration of 10.5 (9.3) years. The education level for most patients $(64.6 \%)$ was secondary school education or less (Table 1). About one-quarter (26.2\%) of patients were in full-time work, $16.0 \%$ were unemployed (and seeking work), and $51.1 \%$ were retired. RA had had a considerable impact on patients' work-life, as $11.9 \%$ had retired early due to RA, while $6.0 \%$ were unemployed due to RA.
Table I Sociodemographic Characteristics

\begin{tabular}{|c|c|}
\hline Characteristics & $\begin{array}{l}\text { Full Analysis } \\
\text { Set }(n=1624)\end{array}$ \\
\hline Female, n (\%) & $1368(84.2)$ \\
\hline Age, years, mean (SD) & $58.4(13.1)$ \\
\hline \multicolumn{2}{|l|}{ Race, n (\%) } \\
\hline White & $143 \mid(88.1)$ \\
\hline Black & $7(0.4)$ \\
\hline Asian & $139(8.6)$ \\
\hline Mixed & $46(2.8)$ \\
\hline Other & $\mathrm{I}(0 . \mathrm{I})$ \\
\hline \multicolumn{2}{|l|}{ Occupation, n (\%) } \\
\hline Employed full-time & $426(26.2)$ \\
\hline Employed part-time (not due to RA) & $28(1.7)$ \\
\hline Employed part-time due to RA & $53(3.3)$ \\
\hline Attending school or university & $12(0.7)$ \\
\hline Unemployed (not due to RA) & $163(10.0)$ \\
\hline Unemployed due to RA & $97(6.0)$ \\
\hline Early retired (not due to RA) & $87(5.4)$ \\
\hline Early retired due to RA & $194(11.9)$ \\
\hline Regularly retired & $549(33.8)$ \\
\hline \multicolumn{2}{|l|}{ Education, n (\%) } \\
\hline No formal education & $27(1.7)$ \\
\hline Primary school & $388(23.9)$ \\
\hline Secondary school (eg high school) & $633(39.0)$ \\
\hline Non-university, professional education & $311(19.2)$ \\
\hline University & $263(16.2)$ \\
\hline \multicolumn{2}{|l|}{ Residence, n (\%) } \\
\hline Urban center (>80,000 inhabitants) & $814(50.1)$ \\
\hline Town (10,000-80,000 inhabitants) & $432(26.6)$ \\
\hline Rural area $(<10,000$ inhabitants $)$ & $378(23.3)$ \\
\hline
\end{tabular}

Abbreviations: RA, rheumatoid arthritis; SD, standard deviation.

Patients had moderate-to-high disease activity, with mean tender and swollen joint counts of 28 joints (TJC28 and SJC28) of 7.8 and 4.6, respectively, and a mean DAS28 with C-reactive protein (CRP) of 4.7 (Table 2). Radiographic structural damage (based on X-ray data $\leq 6$ months from enrollment) was apparent in $76.9 \%$ of patients with valid data $(\mathrm{n}=934)$. Rheumatoid factor (RF) status was positive for $78.4 \%$ of patients with valid data $(\mathrm{n}=1044)$, as was anti-citrullinated protein antibody (ACPA) status for $74.1 \%$ (valid data $n=717$ ), with a total of $82.2 \%$ ( $n=1060$ of patients with valid data $<3$ months from enrollment) of seropositive patients, defined as RF and/or ACPA positive. Most patients had 
Table 2 Rheumatoid Arthritis Disease Activity and Joint Assessment

\begin{tabular}{|c|c|}
\hline Measure, Mean (SD) & Full Analysis Set $(n=1624)^{2}$ \\
\hline TJC28 (0-28) & $7.8(5.4)$ \\
\hline SJC28 (0-28) & $4.6(4.2)$ \\
\hline PtGA (VAS, $0-10 \mathrm{~cm}$ ) & $5.7(2.1)$ \\
\hline PhGA (VAS, $0-10 \mathrm{~cm}$ ) & $5.2(1.9)$ \\
\hline DAS28-CRP & 4.7 (I.I) \\
\hline DAS28-ESR ${ }^{c}$ & $5.0(I . I)$ \\
\hline CDAI (0-76) & $23.3(10.6)$ \\
\hline SDAI $(0-86)^{b}$ & $26.9(12.9)$ \\
\hline
\end{tabular}

Notes: ${ }^{a}$ Unless otherwise stated. ${ }^{b} n=1500$ patients with valid data. ${ }^{c} n=1515$ patients with valid data.

Abbreviations: CDAI, Clinical Disease Activity Index; DAS28-CRP, Disease Activity Score in 28 joints with C-reactive protein; DAS28-ESR, Disease Activity Score in 28 joints with erythrocyte sedimentation rate; PhGA, Physician Global Assessment; PtGA, Patient Global Assessment; SD, standard deviation; SDAl, Simplified Disease Activity Index; SJC28, swollen joint count in 28 joints; TJC28, tender joint count in 28 joints; VAS, visual analog scale.

comorbidities $(73.3 \% ; \mathrm{n}=1191)$, the most common of which were cardiac disorders $(36.6 \% ; n=595)$, musculoskeletal connective tissue disorders $(25.6 \% ; n=415)$, endocrine disorders $(19.8 \% ; \mathrm{n}=322)$, and metabolic/nutrition disorders $(18.5 \% ; n=301)$. Vascular disorders were present in $9.9 \%(n=160)$, and psychiatric disorders in $6.8 \%$ $(\mathrm{n}=111)$ of patients.

Healthcare resource utilization (HRU) was relatively high in this population, with frequent visits to healthcare professionals. Most (98\%; $n=1153)$ of these visits were outpatient visits, although $7.4 \%(n=87)$ of patients had an emergency room visit, and $13.5 \%(n=159)$ had been hospitalized for a mean (SD) length of stay of 7.5 (6.1) days in the 3 months prior to the study. For patients who had had an outpatient or emergency room visit in the 3 months prior to study visit, the mean (SD) number of visits was 2.2 (2.5) and $1.6(1.3)$, respectively.

\section{Medication and Treatment Strategy}

csDMARDs were the most frequently prescribed medications, used by $1447(89.1 \%)$ patients, followed by bDMARDs $(32.6 \% ; \mathrm{n}=530)$ and tsDMARDs $(2.4 \%$; $\mathrm{n}=39$; Table 3). Across all classes of DMARDs, more than half of the patients $(53.9 \% ; n=875)$ received their DMARD as monotherapy. Within each of the DMARD classes, 709/1447 (49.0\%) patients received csDMARDs as monotherapy, $150 / 530(28.3 \%)$ received bDMARDs as
Table 3 Current Medications Administered for Rheumatoid Arthritis

\begin{tabular}{|l|l|}
\hline Medication, $\mathbf{n}(\%)$ & Full Analysis Set (n=1624) \\
\hline csDMARD & $1447(89.1)$ \\
Methotrexate & $991(61.0)$ \\
Leflunomide & $363(22.4)$ \\
Hydroxychloroquine & $244(15.0)$ \\
Sulfasalazine & $212(13.1)$ \\
Chloroquine & $22(1.4)$ \\
\hline bDMARD & $530(32.6)$ \\
Adalimumab & $116(7.1)$ \\
Etanercept & $115(7.1)$ \\
Rituximab & $73(4.5)$ \\
Tocilizumab & $72(4.4)$ \\
Abatacept & $52(3.2)$ \\
Infliximab & $39(2.4)$ \\
Certolizumab pegol & $32(2.0)$ \\
Golimumab & $30(1.8)$ \\
\hline tsDMARD & $39(2.4)$ \\
Tofacitinib & $34(2.1)$ \\
Baricitinib & $5(0.3)$ \\
\hline Systemic glucocorticoids & $163(10.0)$ \\
\hline
\end{tabular}

Abbreviations: bDMARD, biologic DMARD; csDMARD, conventional synthetic DMARD; DMARD, disease-modifying antirheumatic drug; tsDMARD, targeted synthetic DMARD.

monotherapy, and 16/39 (41.0\%) received tsDMARDs as monotherapy. Out of all patients, $21.7 \%(n=352)$ and $2.7 \%$ $(n=44)$ of patients received double or triple csDMARDs, respectively. A total of $61.0 \%(n=991)$ received the anchor drug methotrexate as part of their treatment regime: of those with valid data $(n=929), 54.4 \%$ received 10 to $15 \mathrm{mg} /$ week, while $39.3 \%$ received $>15 \mathrm{mg} /$ week. Only $10.0 \%(n=163)$ of patients used systemic glucocorticoids for RA (FAS).

Only half $(51.8 \% ; n=840)$ of all patients had a planned switch of medication, despite long-standing disease and suboptimal symptom control with current DMARD therapy. Among these patients, a planned switch to a TNFi was most frequently reported (42.4\%; Figure 1$)$.

Regression analysis revealed that predictors for a decision to switch to a different DMARD by the treating physician included younger patient age $(P=0.0012)$, having a high disease activity by DAS28 with erythrocyte sedimentation rate (DAS28-ESR) $(P<0.0001$ for high disease activity $>5.1$ vs moderate/low disease activity $\leq 5.1$ ), worse HAQ-DI physical function $(P=0.0062)$, lower number of current DMARDs $(P<0.0001)$, lower global satisfaction score 


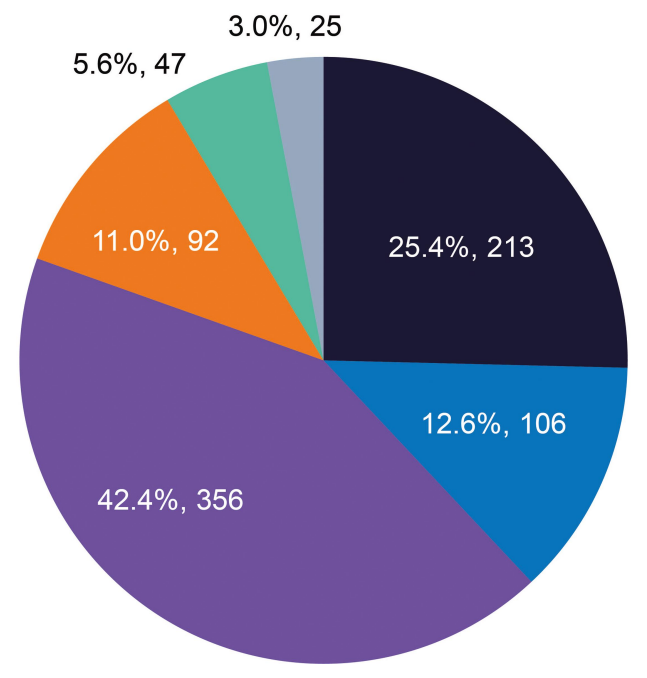

- csDMARD

- tsDMARD: JAK inhibitor

bDMARD: TNF inhibitor

bDMARD: IL-6 inhibitor

bDMARD: B-cell inhibitor

bDMARD: T-cell receptor inhibitor

Figure I Mode of action for planned DMARD switches.

Note: Treatment switch to a different DMARD was planned for 840 patients (51.8\%) out of 1623 patients with valid data.

Abbreviations: bDMARD, biologic DMARD; csDMARD, conventional synthetic DMARD; DMARD, disease-modifying antirheumatic drug; IL-6, interleukin-6; JAK, Janus kinase; TNF, tumor necrosis factor.

$(P<0.0001)$, and patient preference for oral administration $(P=0.0019)$. Predictors for not switching to a different DMARD included a higher number of comorbidities $(P<0.0001)$, lower current DAS28-ESR score $(P<0.0001)$, lower number of concomitant medications $(P=0.0002)$, current treatment with ts/bDMARDs $(P<0.0001)$, and higher TSQM effectiveness subscore $(P<0.0001)$. In addition, physicians in Asia were significantly less likely to switch to a different DMARD than physicians in South America $(P=0.0069)$ or Europe $(P=0.0136)$.

A switch to the recently introduced tsDMARD class was reported in $12.6 \%$ of patients with planned switching and was significantly associated with RA disease duration $\geq 2$ years, DAS28-ESR > 5.1, higher HAQ-DI, and patients' preference for oral administration.

Overall, $61.4 \%$ of FAS patients took any medication for their current comorbidities; the mean (SD) number of medications administered for comorbidities was 2.1 (1.9). The most frequent medications for comorbidities were selective beta blocking agents (12.6\%), vitamin D and analogs (12.0\%), plain angiotensin-converting enzyme inhibitors (10.9\%), and proton pump inhibitors $(10.3 \%)$. Platelet aggregation inhibitors (excluding heparin) were used by $4.2 \%$ of patients.

\section{Primary Outcome: Patients' RA Treatment Satisfaction}

Mean (SD) TSQM global satisfaction subscore was 60.9 (20.9) (Figure 2). Based on a TSQM global satisfaction

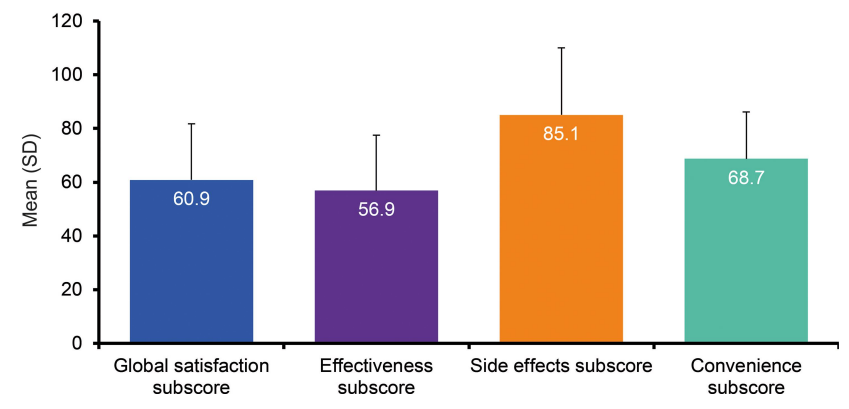

Figure 2 Treatment Satisfaction Questionnaire for Medication subscores. Notes: Full analysis set $(n=1624)$. Each TSQM subscore ranges from 0 to 100, with higher scores denoting greater satisfaction.

Abbreviations: SD, standard deviation; TSQM, Treatment Satisfaction Questionnaire for Medication.

subscore cut-off value of $\geq 80$, as few as $13.5 \%$ of patients reported good treatment satisfaction.

In a subgroup analysis of those treated with advanced therapies (b/tsDMARDs), patients treated with an oral DMARD $(\mathrm{n}=52)$ had significantly lower mean TSQM global satisfaction ( 60.4 vs $68.9, P=0.0212)$ and effectiveness (54.2 vs 63.1, $P=0.0023$ ) subscores compared with patients treated with parenteral DMARD $(\mathrm{n}=517)$. Patients on DMARD monotherapy ( $\mathrm{n}=875$ ) had significantly lower mean TSQM global satisfaction (59.7 vs $62.2, P=0.0262$ ) and effectiveness (55.9 vs 58.0, $P=0.0268$ ) subscores compared with patients using DMARD combination therapy $(\mathrm{n}=749)$.

Multivariate logistic regression analysis indicated that high disease activity (DAS28-ESR >5.1) was a negative predictor of good treatment satisfaction, while current 
Table 4 Predictors of Good Treatment Satisfaction (TSQM Global Satisfaction Score $\geq 80$ )

\begin{tabular}{|l|l|l|l|}
\hline Variables & Effect Estimate & $P$-value & OR (95\% CI) \\
\hline High disease activity (DAS28-ESR >5.I) & -0.6045 & 0.0026 & $0.546(0.368,0.810)$ \\
\hline SF-36 PCS & 0.0554 & $<0.0001$ & $1.057(1.036,1.078)$ \\
\hline SF-36 MCS & 0.0340 & $<0.0001$ & $1.035(1.019,1.050)$ \\
\hline Psychiatric disorders & 0.9475 & 0.0004 & $2.579(1.531,4.344)$ \\
\hline Current treatment with ts/bDMARDs & 1.2665 & $<0.0001$ & $3.548(2.593,4.856)$ \\
\hline
\end{tabular}

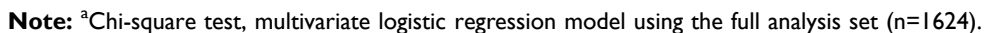

Abbreviations: bDMARD, biologic DMARD; Cl, confidence interval; DAS28-ESR, Disease Activity Score in 28 joints with erythrocyte sedimentation rate; DMARD, disease-modifying antirheumatic drug; MCS, Mental Component Summary; OR, odds ratio; PCS, Physical Component Summary; SF-36, 36-item Short Form Health Survey; tsDMARD, targeted synthetic DMARD; TSQM, Treatment Satisfaction Questionnaire for Medication.

treatment with ts/bDMARD, higher SF-36 PCS and SF-36 MCS scores, and the presence of a psychiatric disorder (not further specified) were positive predictors of good treatment satisfaction (Table 4).

\section{Secondary Outcomes: Other PROs}

RA was shown to have an impact on measures of HRQoL and work productivity, as shown in Table 5. Work ability showed significant impairment: among patients who were employed $(n=413)$, the median total work productivity impairment score was 50, representing a 50\% work productivity loss because of RA. Similarly, patients reported $60 \%$ total activity impairment (Table 5).
Of the 1601 patients with valid data, mean (SD) total eHEALS score was 21.3 (8.4). Poor DHL, defined as eHEALS $<26$, was evident in two-thirds of patients $(67.4 \% ; n=1079)$. In general, $35.4 \%$ of patients found the internet "not useful" or "not useful at all" in helping to inform decisions about their own health, 33.2\% found it "useful" or "very useful", and 31.4\% were unsure of its impact. A total of $38.6 \%$ of patients believed it is "important" or "very important" to be able to access health resources on the internet, $36.9 \%$ considered it as "not important" or "not important at all", and $24.5 \%$ were unsure.

A total of 1623 patients reported their adherence to medication; most (87.4\%) reporting good adherence, defined as

Table 5 Patient-Reported Outcomes

\begin{tabular}{|c|c|c|}
\hline Patient-Reported Outcome & $\mathbf{n}^{\mathbf{a}}$ & Mean (SD) ${ }^{b}$ \\
\hline Worst joint pain $(0-10)$ & 1623 & $6.0(2.5)$ \\
\hline Severity of morning stiffness $(0-10)$ & 1623 & $5.0(2.8)$ \\
\hline Duration of morning stiffness, hours, median (IQR) & 1488 & $1.0(0.5-2.0)$ \\
\hline FACIT-F score $(0-52)$ & 1623 & $28.1(11.2)$ \\
\hline HAQ-DI score $(0-3)$ & 1624 & I.3 (0.7) \\
\hline SF-36 PCS $(0-100)$ & 1623 & $36.6(7.9)$ \\
\hline SF-36 MCS $(0-100)$ & 1623 & $41.9(11.0)$ \\
\hline \multicolumn{3}{|l|}{ WPAI-RA scores, \%, median (IQR) } \\
\hline Presenteeism ${ }^{c}$ & 418 & $40.0(20.0-70.0)$ \\
\hline Absenteeism ${ }^{c}$ & 461 & $0.0(0.0-16.7)$ \\
\hline Total work productivity impairment ${ }^{c}$ & 413 & $50.0(20.0-70.0)$ \\
\hline Total activity impairment & 1619 & $60.0(30.0-80.0)$ \\
\hline
\end{tabular}

Notes: ${ }^{a}$ Valid data from the full analysis set $(n=1624)$. 'Unless otherwise stated. 'Employed patients only.

Abbreviations: FACIT-F, Functional Assessment of Chronic Illness Therapy-Fatigue; HAQ-DI, Health Assessment Questionnaire-Disability Index; IQR, interquartile range; MCS, Mental Component Summary; PCS, Physical Component Summary; SD, standard deviation; SF-36, 36-item Short Form Health Survey; WPAI-RA, Work Productivity and Activity Impairment-Rheumatoid Arthritis. 


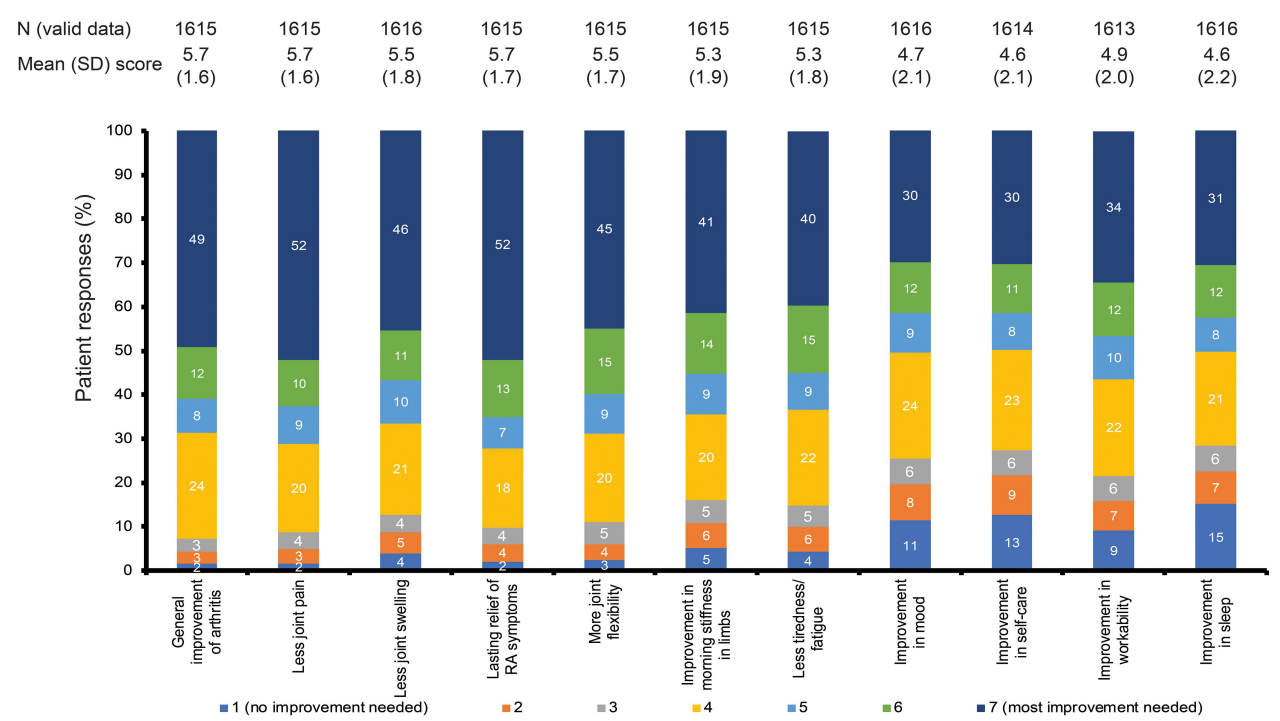

Figure 3 Patients' expectations for RA treatments.

Notes: Patients scored their expectations based on a 7-point numeric rating scale where $\mathrm{I}=$ no improvement needed to $7=$ the most improvement needed. The mean (SD) score and distribution of scores are shown for each treatment expectation. Due to rounding, percentages may not sum to $100 \%$.

Abbreviations: RA, rheumatoid arthritis; SD, standard deviation.

$\geq 80 \%$ on the VAS. Mean (SD) self-reported adherence was $91.4 \%$ (16.9). After correcting for confounders, the level of self-reported adherence was positively associated with TSQM global satisfaction subscore $(P=0.0059)$ and with lower levels of joint pain $(P=0.0473)$. There was no significant association with the other variables evaluated in the model (Supplementary Table 2).

\section{PPI Outcomes: Patient Expectations for Pharmacologic Treatment}

When asked about treatment expectations, patients rated "general improvement of arthritis", "less joint pain", and "lasting relief of RA symptoms" highest for their treatment expectations, with mean (SD) scores of 5.7 (1.6-1.7) for each (Figure 3). The strongest association between the top three highest-rated treatment expectations and outcomes was worst joint pain ( $\rho=0.31, \rho=0.35$, and $\rho=0.31$, respectively; all $P<0.0001)$.

\section{PPI Outcomes: Patient Preferences for Pharmacologic Treatment}

Most patients preferred oral administration of medication $(60.7 \%)$ and rapid $(\leq 1$ week) onset of treatment action (71.1\%) (Figure 4). After correcting for confounders, patient preference for oral administration was significantly associated with older age $(P=0.0005)$, duration of RA $<2$ years $(P<0.0001)$, and a greater number of comorbidities $(P=0.0014)$, however, we did not find any significant relationships with the other variables evaluated in the model (Supplementary Table 2).

While $37.4 \%$ of patients felt treatment requiring combination with another drug once a week was acceptable (Figure 4), 31.3\% of patients preferred not to use drug combinations for RA. After correcting for confounders, patient preference for monotherapy was significantly associated with a lower number of current DMARDs $(P=0.0054)$ and lower TSQM global satisfaction subscore $(P<0.0001)$. We did not find any significant association with the other variables evaluated in the model (Supplementary Table 2).

In terms of potential side effects of RA therapies, patients indicated that the "most acceptable" side effect was "weight gain", while "increased risk for malignancies" and "increased risk for cardiovascular disease" were the "least acceptable" (Figure 4).

\section{PPI Outcomes: Need for PSP}

When patients were asked about the need for PSPs, patients assigned the greatest importance for educational materials about RA disease and therapy, followed by the need for a PSP in general (Figure 5). Conversely, the need for appointment reminders, digital/lifestyle interventions, and medication administration reminders were rated as least important. No strong correlations were found for the need for PSP and other variables. 


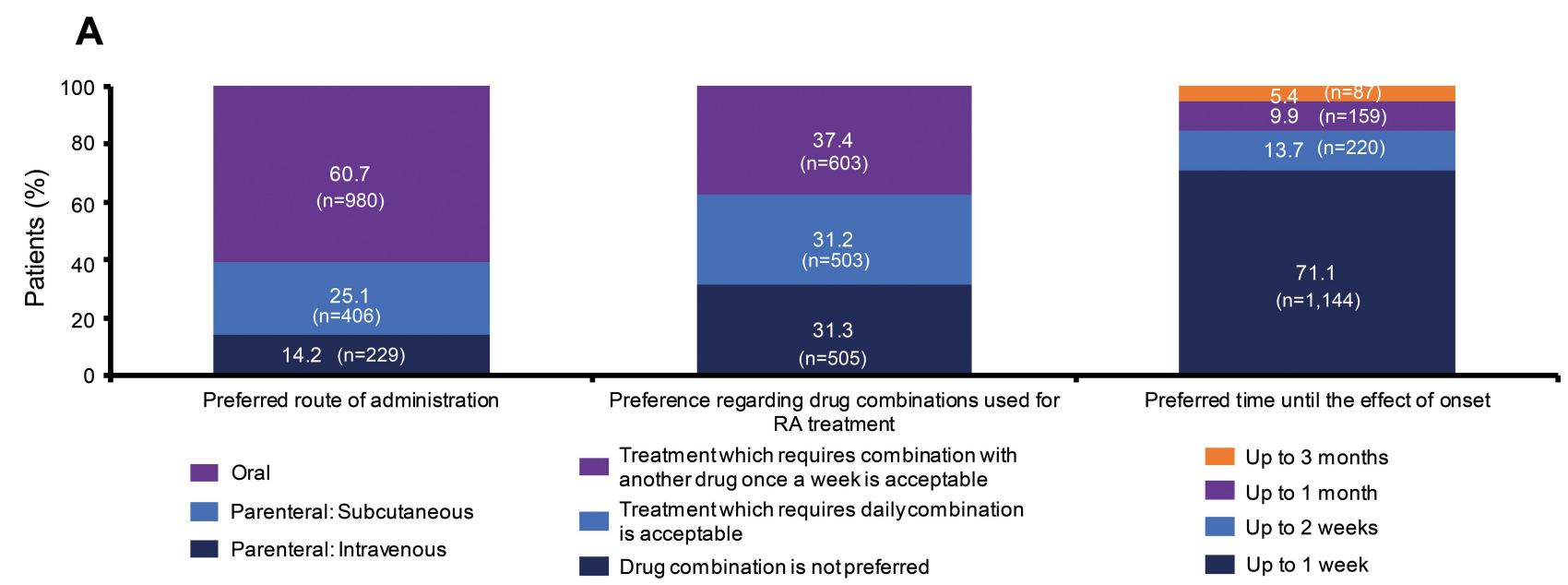

\section{B}

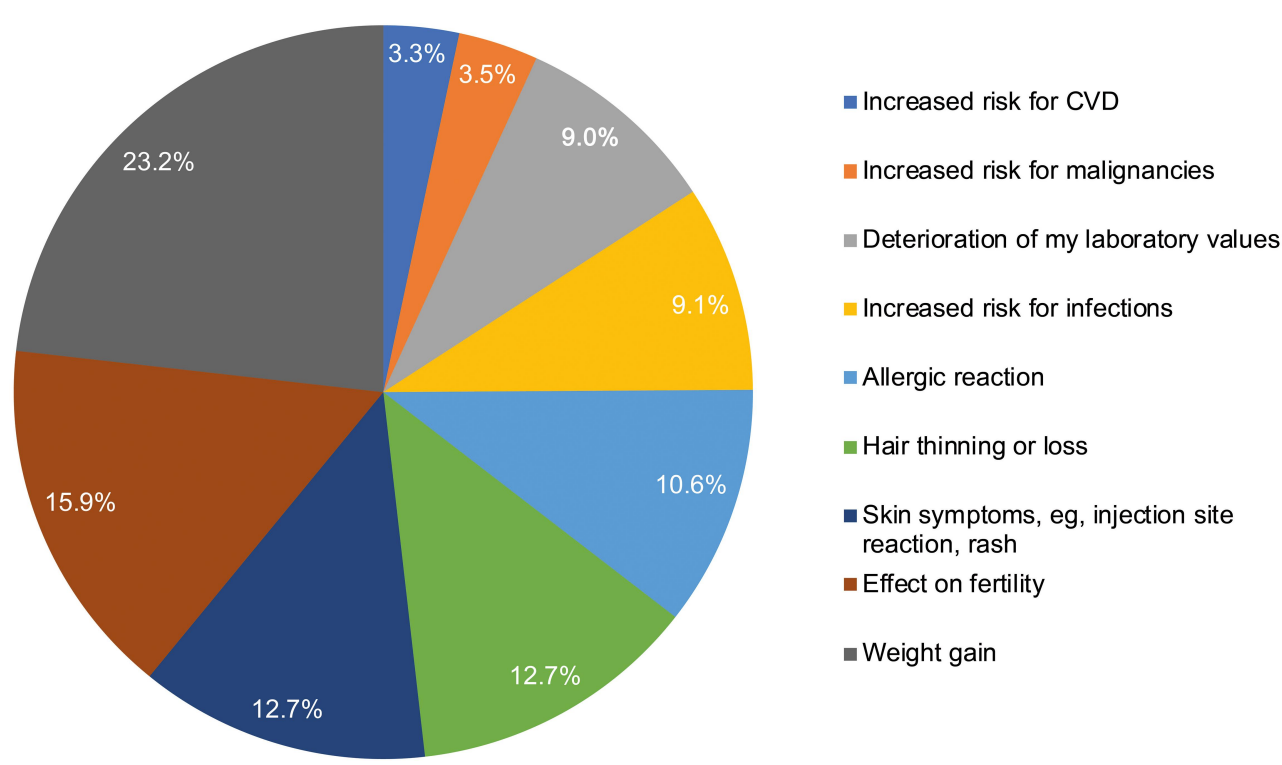

Figure 4 Patients' preferences for RA treatments: (A) route of administration, drug combinations, and time to effect; (B) acceptability of potential side effects of RA treatments.

Note: $(B) n=1608$

Abbreviations: CVD, cardiovascular disease; RA, rheumatoid arthritis.

\section{Discussion}

In this large international study of patients with poorly controlled RA despite DMARD treatment, only $13.5 \%$ of patients reported good treatment satisfaction as measured by a validated tool, despite $87.4 \%$ of patients reporting good treatment adherence. Only half of these patients had a planned switch of medication despite suboptimal disease control, impairments in HRQoL and work ability, and high levels of HRU. We found that patients preferred treatments that were administered orally and/or had a rapid onset of action; sustained and general improvement of RA and improvement in joint pain were the leading treatment expectations.
The low treatment satisfaction scores reported in this international population of patients with suboptimal disease control was consistent with previous findings specific to a single country (USA and Germany). ${ }^{9,15}$ In a multivariate model, we identified high vs low disease activity as a negative predictor of good treatment satisfaction, whereas better HRQoL scores and administration of advanced treatment (b/tsDMARD) were positive predictors of good treatment satisfaction. The positive impact of treatment with b/tsDMARDs on patient satisfaction was consistent with previous studies in Germany and Japan, which included patients with RA irrespective of disease control. ${ }^{9,28}$ Consistent with our findings, the German study also reported a positive correlation between treatment 


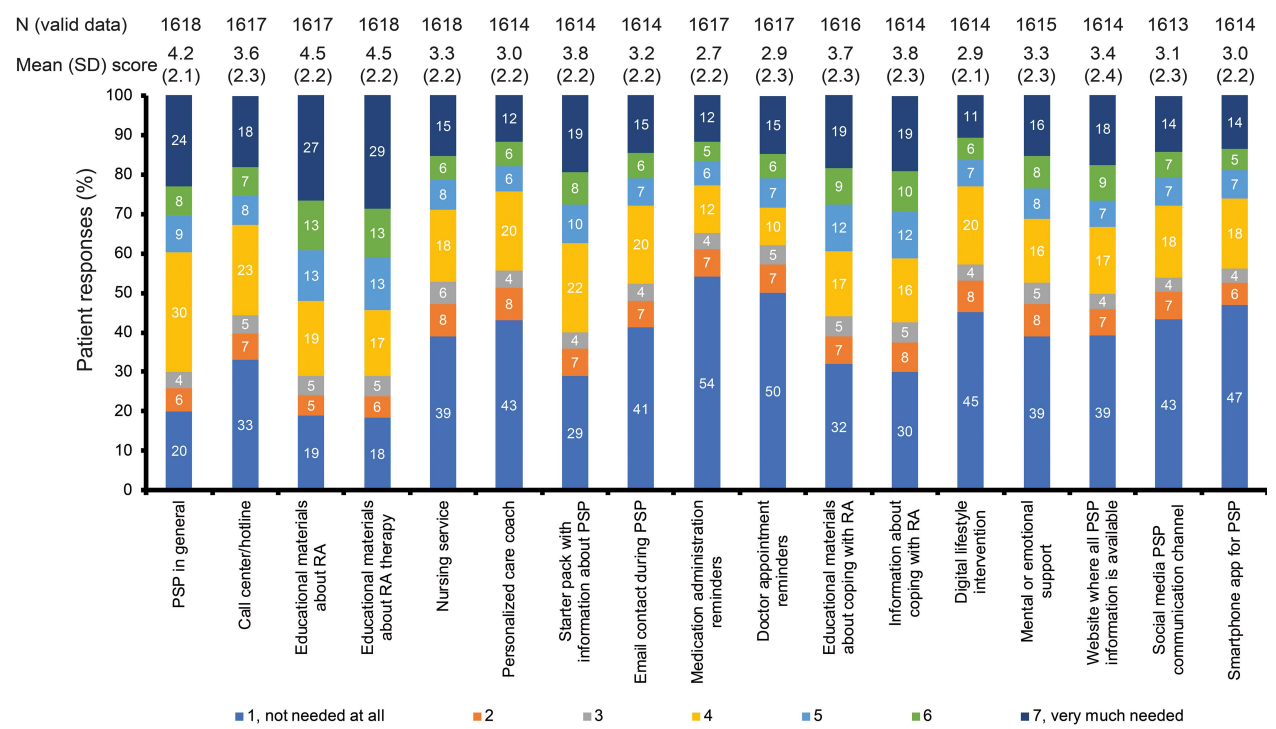

Figure 5 Patients' need for patient support programs.

Notes: Patients scored their perceived need for PSP items based on a 7-point numeric rating scale where I = not needed at all to $7=$ very much needed. Patients received a short description of PSPs as part of the questionnaire. The mean (SD) score and distribution of scores are shown for each PSP item. Due to rounding, percentages may not sum to $100 \%$.

Abbreviations: PSP, patient support program; RA, rheumatoid arthritis; SD, standard deviation.

satisfaction and improved disease activity and/or physical function. ${ }^{9}$ Of note, we also found a positive association between good treatment satisfaction and the presence of psychiatric disorders. This could imply that a perceived good articular response to therapy may be associated with improving mental state, or that those with mental health disorders are less discerning with respect to expectations of therapeutic response; further research is needed to confirm these hypotheses. Previous reports have suggested that treatment satisfaction may be influenced by the mental state of patients although, contrary to our findings, patients with personality disorders and depression, in general $^{29,30}$ or in a rheumatology setting, 9,28 experienced lower levels of treatment satisfaction. However, these results must be interpreted with caution, because the specific psychiatric diagnoses were not captured in this study. Moreover, these results do not rule out the influence of other unknown confounding factors, as satisfaction can include other aspects that were not evaluated in our study such as level and access to service, changes in disease activity during the particular therapy, unmet expectations, patient-physician relationship, and patients' beliefs and concerns. ${ }^{10}$ Assessing and understanding these patient characteristics can help toward identifying patients who may be unwilling to change medication despite suboptimal control. ${ }^{31}$

Study patients had poorly controlled RA with moderate-to-high disease activity for 1 to 4 months despite full tolerable dosages of DMARD therapy, which should trigger treatment adjustment under a T2T strategy. For such patients, RA guidelines recommend that treatment should be adjusted, ${ }^{32,33}$ yet $>40 \%$ of patients were still receiving a csDMARD as monotherapy rather than adding or switching to a $b /$ tsDMARD or adding a second csDMARD to therapy. A switch to a different DMARD was planned for only half of the patients and, of those patients who planned a switch, only three-quarters considered stepping up to advanced therapies with a bDMARD or tsDMARD. Although some of these treatment decisions may have been driven by reimbursement restrictions in participating countries with limited healthcare resources, this failure to plan treatment switches despite inadequate disease control suggests a degree of "treatment inertia". Other real-world data indicated that many patients with poorly controlled RA remain on their current regimens rather than escalating therapy. ${ }^{34}$ T2T approaches can be demanding for patients and physicians: some patients may not be willing to commit to more frequent and necessary clinic visits, while others may not have easy access to rheumatologists. ${ }^{35}$ Furthermore, as non-rheumatologists have limited experience with and confidence in administering DMARD therapy, treatment escalation is rarely performed outside of the rheumatology setting. Therefore, more support should be provided to prevent treatment inertia, eg, through patient 
education and by raising awareness of the importance of T2T approaches with physicians.

In this study, high disease activity defined by DAS28ESR $>5.1$ predicted the decision to switch treatments, while a lower DAS28-ESR score predicted the decision not to switch. A recent study has found that DAS28-ESR is associated with a numerically higher value than DAS28CRP; such that using 5.1 as an absolute cut-off for DAS28-CRP may underestimate the number of patients with high disease activity. ${ }^{36}$ Therefore, the distinction between DAS28-ESR and DAS28-CRP as measures of disease activity should be considered, especially when treatment access is dependent on DAS28 disease activity.

Adherence to therapy is essential if patients are to gain the most benefit from their treatments. In the current study, almost $90 \%$ of patients reported good adherence to their medication. This is consistent with the upper limits of adherence observed in the international ALIGN study $(57-76 \%)^{37}$ and in a systematic review of US studies (16-81\% with TNFis including etanercept, adalimumab, and infliximab). ${ }^{38}$ The high adherence rate in the current study suggests that suboptimal disease control in this population is unlikely to be due to poor adherence, although there may be a possibility of ascertainment or response bias. We also found that treatment adherence was significantly associated with treatment satisfaction and lower levels of pain, one of the leading patient expectations identified in this study. This finding underlines the importance of effective pain control in RA.

Although the SENSE population largely comprised older persons, with only $26.2 \%$ in full-time employment, suboptimally controlled RA had a substantial impact on working life: Of those employed, based on responses to the WPAI-RA questionnaire, $50 \%$ of total work productivity loss and $40 \%$ of presenteeism were attributable to RA. The levels of impairment in SENSE appear greater than the findings from a previous cross-sectional study in a similar setting using the same validated tool (WPAIRA). ${ }^{39}$ Reduced work productivity is associated with poor HRQoL driven by greater disease activity, worse mental health, and greater physical disability and pain. $^{40,41}$ The impact of worsening disease can be profound, so it is important that a strategy to achieve the tightest disease control possible is adopted to preserve work ability and decrease the indirect societal cost of the disease.

Consistent with previous research, most patients in SENSE preferred oral monotherapy, ${ }^{3,16,25}$ largely because of convenience and ease of use. Significant predictors for oral preference included older age, shorter disease duration, and a greater number of comorbidities. Although many patients were open to combination therapy, $31 \%$ preferred not to use drug combinations, and the latter preference was associated with a lower current number of DMARDs and a lower treatment satisfaction. It has been suggested that psychosocial factors, such as education, may be associated with a belief in a stronger treatment effect being associated with parenteral and/or combination therapies, but our data do not support this theory.

When considering patient expectations, the highestrated treatment expectations related to treatment benefit: improvement of arthritis, less joint pain, and lasting relief of symptoms. This is consistent with the findings of a systematic review of treatment preferences in which patients with RA placed most importance on treatment benefit over other treatment attributes, including serious or minor side effects and cost or route of administration. ${ }^{14}$ However, patient preferences are variable and mutable, so understanding patient preferences on an individual level is likely to aid shared decision-making between patients and their physicians.

Patient concerns relating to side effects are of growing importance, especially as patients' beliefs about harms associated with medications are significant predictors of adherence in patients with RA. ${ }^{37}$ Previous reports have suggested that patients were more willing to accept side effects associated with temporary cosmetic changes, such as weight gain or allergic reactions, and least willing to take medications associated with deterioration of laboratory values, infections, or a risk of major toxicity such as cancer. ${ }^{42}$ Similar findings were evident in SENSE, with weight gain being the most acceptable side effect reported by patients, whereas increased risk of malignancies or cardiovascular disease were the least acceptable. A clear understanding of the most acceptable side effects can support shared decision-making at the individual level, as well as the development of new medications at the population level.

Patients recognized a need for a PSP in general, although reminders and digital/lifestyle interventions were considered less important. Adoption of eHealth technologies may be a key strategy in supporting and providing care for patients with chronic diseases, such as RA, and good DHL is a prerequisite for the optimum use of eHealth tools. Among the SENSE patient population, twothirds had poor DHL, and only one-third found the internet 
important or useful in helping them access health resources or make decisions about their own health, which may be a reflection of the relatively advanced age of the population. Other studies have reported poor DHL, highlighting a need to assist patients with poor DHL, so they may realize the potential benefits of eHealth tools as part of PSPs. $^{43,44}$

There are several limitations to this study. Noninterventional studies have inherent limitations on data quality compared with randomized controlled trials. For instance, patients were selected based on convenience (resulting in potential selection bias) and willingness to complete time-consuming PRO questionnaires, with all PROs having inherent response and assessment bias. Likewise, patient-reported HRU is prone to recall bias. PPI questionnaires used in the study have not been validated, which may limit their reliability and generalizability. Furthermore, while the direct preference assessment method used for the PPI questionnaires is considered a valid tool to determine treatment characteristics that are important to patients, it does not provide information about the degree of importance of these characteristics and the trade-offs patients are willing to make. ${ }^{16}$ Data from this observational study portray a diverse, multinational study cohort under real-world conditions. Country-specific analyses are warranted to determine the impact of local culture, and healthcarerelated and socioeconomic factors on patient outcomes. Patient selection criteria limit the generalizability of results across all RA patient populations, as often patients with better disease control and shorter duration of disease are recruited for studies. However, this study provides important insight into the proportion of patients with RA who are in need of treatment adjustment, both in line with recent treatment standards and taking into account T2T principles.

\section{Conclusion}

The findings from SENSE demonstrate that suboptimal disease control has a significant impact on patients' treatment satisfaction, working life, and HRU. Patient preferences and attitudes may influence treatment satisfaction and adherence to DMARDs, which ultimately will impact real-world efficacy. Poor DHL may represent an obstacle to patients' understanding of information critical to decision-making. Taken together, these results support the commitment to current T2T strategies, and they also highlight the critical need to individualize treatment decision-making.

\section{Abbreviations}

ACPA, anti-citrullinated protein antibody; ACR, American College of Rheumatology; bDMARD, biologic DMARD; CDAI, Clinical Disease Activity Index; CRP, C-reactive protein; CVD, cardiovascular disease; csDMARD, conventional synthetic DMARD; DAS28, Disease Activity Score in 28 joints; DHL, digital health literacy; DMARD, disease-modifying antirheumatic drug; eHEALS, eHealth Literacy Scale; ESR, erythrocyte sedimentation rate; EULAR, European League Against Rheumatism; FACIT-F, Functional Assessment of Chronic Illness Therapy-Fatigue; FAS, full analysis set; HAQ-DI, Health Assessment Questionnaire-Disability Index; HRQoL, health-related quality of life; HRU, healthcare resource utilization; IQR, interquartile range; MCS, Mental Component Summary; NRS, numeric rating scale; PCS, Physical Component Summary; PhGA, Physician Global Assessment; PPI, patient preference information; PRO, patient-reported outcome; PSP, patient support program; PtGA, Patient Global Assessment; QoL, Quality of Life; RA, rheumatoid arthritis; RF, rheumatoid factor; SD, standard deviation; SDAI, Simplified Disease Activity Index; SF-36, 36-item Short Form Health Survey; SJC28, swollen joint count of 28 joints; T2T, treat-to-target; TJC28, tender joint count of 28 joints; TNFi, tumor necrosis factor inhibitor; tsDMARD, targeted synthetic DMARD; TSQM, Treatment Satisfaction Questionnaire for Medication; VAS, visual analog scale; WPAI, Work Productivity and Activity Impairment.

\section{Data Sharing Statement}

AbbVie is committed to responsible data sharing regarding the clinical trials we sponsor. This includes access to anonymized, individual, and trial level data (analysis datasets), as well as other information (eg, protocols and Clinical Study Reports), provided the trials are not part of an ongoing or planned regulatory submission. This includes requests for clinical trial data for unlicensed products and indications. These clinical trial data can be requested by any qualified researchers who engage in rigorous, independent scientific research and will be provided following review and approval of a research proposal and statistical analysis plan and execution of a Data Sharing Agreement. Data requests can be submitted at any time, and the data will be accessible for 12 months, with possible extensions considered. For more information on the process, or to submit a request, 
visit https://www.abbvie.com/our-science/clinical-trials/ clinical-trials-data-and-information-sharing/data-andinformation-sharing-with-qualified-researchers.html.

\section{Ethics Approval}

The study was conducted in accordance with the Declaration of Helsinki and has been approved by local ethics committees according to local laws and regulations for participating countries (Supplementary Table 1). All patients provided written authorization to the investigator to use/disclose personal and/or health data or to provide informed consent if required by local regulations.

\section{Acknowledgments}

AbbVie and the authors thank all study investigators for their contributions and the patients who participated in these studies. PCT thanks the National Institute of Health Research for their funding of The NIHR Biomedical Research Centre in Musculoskeletal Disease at Oxford University Hospitals NHS Trust and the University of Oxford. Medical writing assistance was provided by Gregory Bezkorovainy (New York, USA) and Hilary Wong (Cheshire, UK) of 2 the Nth and was funded by AbbVie.

\section{Author Contributions}

All authors made substantial contributions to the conception and design, acquisition of data, or analysis and interpretation of data; took part in drafting the article or revising it critically for important intellectual content; agreed to submit to the current journal; gave final approval of the version to be published; and agree to be accountable for all aspects of the work.

\section{Funding}

AbbVie funded this study and participated in the study design, research, analysis, data collection, interpretation of data, review, and approval of the publication. All authors had access to relevant data and participated in the drafting, review, and approval of this publication. No honoraria or payments were made for authorship.

\section{Disclosure}

Peter C. Taylor received research grants, consultation, and/ or speaking fees from AbbVie, Biogen, Bristol-Myers Squibb, Celgene, Celltrion, Fresenius, Galapagos, Gilead, GSK, Janssen, Eli Lilly, Sanofi, Nordic Pharma, Novartis, Pfizer, Roche, and UCB; Codrina Ancuta received consultation and/or speaker fees from AbbVie, Eli Lilly, Ewopharma, Merck Sharpe and Dohme, Novartis, Pfizer, Roche, and UCB; Andrey Gordeev received speaker fees from AbbVie; Radka Janková received speaker fees from AbbVie, Bristol-Myers Squibb, Eli Lilly, Merck Sharpe and Dohme, Pfizer, Roche, Sanofi, and UCB; Umut Kalyoncu received consultation and/or speaker fees from AbbVie, Amgen, Eli Lilly, Janssen, Novartis, Pfizer, Roche, and UCB; Jadranka Morović-Vergles received speaker fees from AbbVie, Eli Lilly, Ewopharma, Merck Sharpe and Dohme, Novartis, Pfizer, Roche, and UCB; Mariana Peixoto G. U. e Silva de Souza received research grants from AbbVie, Bristol-Myers Squibb, GSK, Pfizer, and UCB; honoraria for consultancies and/or speaking engagements from AbbVie, Janssen, Pfizer, Roche, and UCB; and is a Member of RA commission - Brazilian Society of Rheumatology and vice-president of SMR (Regional Society of Rheumatology); Prodromos Sidiropoulos received research grants, consultation, and/or speaking fees from AbbVie, Amgen, MSD, Novartis, Pfizer, Roche, and UCB; Atsushi Kawakami received speaker fees from AbbVie GK., Actelion Pharmaceuticals Japan Ltd., Asahi Kasei Pharma Corporation., Astellas Pharma Inc, Celltrion Healthcare Co., Ltd., Chugai Pharmaceutical Co., Daiichi Sankyo Co., Eisai Co., Eli Lilly Japan, GlaxoSmithKline K. K., Janssen Pharmaceutical K.K., Kowa Co., Ltd., MedPeer Inc, Mitsubishi Tanabe Pharma Co., Novartis Pharma Inc, ONO Pharmaceutical Co., Taisho Pharma Co., Ltd., Takeda Pharmaceutical Co., Ltd., and Pfizer Japan Inc; and grants and research support from AbbVie GK., Actelion Pharmaceuticals Japan Ltd., Asahi Kasei Pharma Corporation, Astellas Pharma Inc, AYUMI Pharmaceutical Co., Boehringer Ingelheim Japan, Bristol-Myers Squibb, Celltrion Healthcare Co., Ltd., Chugai Pharmaceutical Co., Daiichi Sankyo Co., Eisai Co., Eli Lilly Japan, Kyowa Hakko Kirin Co., MSD K.K., Neopharma Japan Co., Ltd., Novartis Pharma Inc, ONO Pharmaceutical Co., Sanofi K. K., Taisho Pharmaceutical Co., Ltd., Takeda Science Foundation, and Teijin Pharma Co. Orsolya Nagy and Ivan Lagunes-Galindo are employees of AbbVie and may own stock or options in AbbVie. No relevant conflicts of interest were declared by María C. de la Vega and Bernadette Rojkovich. The authors report no other conflicts of interest related to this work.

Some of the data from this paper were presented at the EULAR 2020 Congress as a poster presentation (SAT0123). The poster's abstract was published in the 
"Scientific Abstracts" section in Annals of Rheumatic Diseases (Taylor et al Ann Rheum Dis 2020; 79:996-997; https://ard.bmj.com/content/79/Suppl_1/996).

\section{References}

1. Yu C, Jin S, Wang Y, et al. Remission rate and predictors of remission in patients with rheumatoid arthritis under treat-to-target strategy in real-world studies: a systematic review and meta-analysis. Clin Rheumatol. 2019;38(3):727-738. doi:10.1007/s10067-018-4340-7

2. Batko B, Batko K, Krzanowski M, Żuber Z. Physician adherence to treat-to-target and practice guidelines in rheumatoid arthritis. J Clin Med. 2019;8:9. doi:10.3390/jcm8091416

3. Louder AM, Singh A, Saverno K, et al. Patient preferences regarding rheumatoid arthritis therapies: a conjoint analysis. Am Health Drug Benefits. 2016;9(2):84-93.

4. Ho MP, Gonzalez JM, Lerner HP, et al. Incorporating patient-preference evidence into regulatory decision making. Surg Endosc. 2015;29(10):2984-2993. doi:10.1007/s00464-014-4044-2

5. FDA guidance: guidance on patient preference information in premarket approval applications, humanitarian device exemption applications, and de novo requests. Available from: http://www.fda.gov/ downloads/medicaldevices/deviceregulationandguidance/guidancedo cuments/ucm446680.pdf. Accessed August 24, 2016

6. FDA guidance: patient preference study design - qualitative steps first steps for sponsors initiating a patient preference study. Available from: https://mdic.org/wp-content/uploads/2016/04/Patient-

Preference-Study-Design-20171102.pdf. Accessed November 2, 2017

7. Marengo MF, Suarez-Almazor ME. Improving treatment adherence in patients with rheumatoid arthritis: what are the options? Int J Clin Rheumatol. 2015;10(5):345-356. doi:10.2217/ijr.15.39

8. Papadimitropoulos M, Mysler E, Garcia E, Lobosco S, Botello B, Leonardi Reyes F. Treatment patterns and satisfaction for rheumatoid arthritis patients in Latin America undergoing advanced therapy. Value Health. 2017;20(9):PA891.

9. Schäfer M, Albrecht K, Kekow J, et al. Factors associated with treatment satisfaction in patients with rheumatoid arthritis: data from the biological register RABBIT. RMD Open. 2020;6:3. doi:10.1136/rmdopen-2020-001290

10. Jackson JL, Chamberlin J, Kroenke K. Predictors of patient satisfaction. Soc Sci Med. 2001;52(4):609-620. doi:10.1016/S02779536(00)00164-7

11. Smolen JS, Aletaha D. What should be our treatment goal in rheumatoid arthritis today? Clin Exp Rheumatol. 2006;24(6 Suppl 43): S-7-13.

12. Van den Bosch F, Ostor AJK, Wassenberg S, et al. Impact of participation in the adalimumab (humira) patient support program on rheumatoid arthritis treatment course: results from the passion study. Rheumatol Ther. 2017;4(1):85-96. doi:10.1007/s40744-0170061-7

13. Norman CD, Skinner HA. eHealth literacy: essential skills for consumer health in a networked world. J Med Internet Res. 2006;8(2):e9. doi:10.2196/jmir.8.2.e9

14. Durand C, Eldoma M, Marshall DA, Bansback N, Hazlewood GS. Patient preferences for disease-modifying antirheumatic drug treatment in rheumatoid arthritis: a systematic review. J Rheumatol. 2020;47(2):176-187. doi:10.3899/jrheum.181165

15. Radawski C, Genovese MC, Hauber B, et al. Patient perceptions of unmet medical need in rheumatoid arthritis: a cross-sectional survey in the USA. Rheumatol Ther. 2019;6(3):461-471. doi:10.1007/ s40744-019-00168-5

16. Alten R, Krüger K, Rellecke J, et al. Examining patient preferences in the treatment of rheumatoid arthritis using a discrete-choice approach. Patient Prefer Adherence. 2016;10:2217-2228. doi:10.2147/PPA.S117774
17. Webster K, Cella D, Yost K. The Functional Assessment of Chronic Illness Therapy (FACIT) Measurement System: properties, applications, and interpretation. Health Qual Life Outcomes. 2003;1:79. doi:10.1186/1477-7525-1-79

18. Fries JF, Spitz P, Kraines RG, Holman HR. Measurement of patient outcome in arthritis. Arthritis Rheum. 1980;23(2):137-145. doi:10.1002/art.1780230202

19. Ware JE Jr, Sherbourne CD, The MOS 36-item short-form health survey (SF-36). I. Conceptual framework and item selection. Med Care. 1992;30(6):473-483. doi:10.1097/00005650-199206000-00002

20. Zhang W, Bansback N, Boonen A, Young A, Singh A, Anis AH. Validity of the work productivity and activity impairment questionnaire-general health version in patients with rheumatoid arthritis. Arthritis Res Ther. 2010;12(5):R177. doi:10.1186/ar3141

21. Walsh JC, Mandalia S, Gazzard BG. Responses to a 1 month self-report on adherence to antiretroviral therapy are consistent with electronic data and virological treatment outcome. Aids. 2002;16 (2):269-277. doi:10.1097/00002030-200201250-00017

22. De Cuyper E, De Gucht V, Maes S, Van Camp Y, De Clerck LS. Determinants of methotrexate adherence in rheumatoid arthritis patients. Clin Rheumatol. 2016;35(5):1335-1339. doi:10.1007/ s10067-016-3182-4

23. Karve S, Cleves MA, Helm M, Hudson TJ, West DS, Martin BC. Good and poor adherence: optimal cut-point for adherence measures using administrative claims data. Curr Med Res Opin. 2009;25 (9):2303-2310. doi:10.1185/03007990903126833

24. Richtering SS, Hyun K, Neubeck L, et al. eHealth literacy: predictors in a population with moderate-to-high cardiovascular risk. JMIR Hum Factors. 2017;4(1):e4. doi:10.2196/humanfactors.6217

25. Fayad F, Ziade NR, Merheb G, et al. Patient preferences for rheumatoid arthritis treatments: results from the national cross-sectional LERACS study. Patient Prefer Adherence. 2018;12:1619-1625. doi:10.2147/PPA.S168738

26. Marshall D, Bridges JF, Hauber B, et al. Conjoint analysis applications in health - how are studies being designed and reported?: an update on current practice in the published literature between 2005 and 2008. Patient. 2010;3(4):249-256. doi:10.2165/11539650000000000-00000

27. Jobanputra P, Maggs F, Deeming A, et al. A randomised efficacy and discontinuation study of etanercept versus adalimumab (RED SEA) for rheumatoid arthritis: a pragmatic, unblinded, non-inferiority study of first TNF inhibitor use: outcomes over 2 years. BMJ Open. 2012;2:6. doi:10.1136/bmjopen-2012-001395

28. Mahlich J, Schaede U, Sruamsiri R. Shared decision-making and patient satisfaction in Japanese rheumatoid arthritis patients: a new "preference fit" framework for treatment assessment. Rheumatol Ther. 2019;6(2):269-283. doi:10.1007/s40744-019-0156-4

29. Hueston WJ, Mainous AG 3rd, Schilling R. Patients with personality disorders: functional status, health care utilization, and satisfaction with care. J Fam Pract. 1996;42(1):54-60.

30. Wyshak G, Barsky A. Satisfaction with and effectiveness of medical care in relation to anxiety and depression. Patient and physician ratings compared. Gen Hosp Psychiatry. 1995;17(2):108-114. doi:10.1016/0163-8343(94)00097-W

31. Stark JL, Yassine M, Nowell WB, et al. THU0159 Barriers to rheumatoid arthritis treatment optimisation: real-world data from the arthritispower registry. Ann Rheum Dis. 2018;77(Suppl 2):299.

32. Singh JA, Saag KG, Bridges SL Jr, et al. 2015 American College of rheumatology guideline for the treatment of rheumatoid arthritis. Arthritis Care Res (Hoboken). 2016;68(1):1-25. doi:10.1002/ acr. 22783

33. Smolen JS, Landewé RBM, Bijlsma JWJ, et al. EULAR recommendations for the management of rheumatoid arthritis with synthetic and biological disease-modifying antirheumatic drugs: 2019 update. Ann Rheum Dis. 2020;79(6):685-699. doi:10.1136/annrheumdis-2019216655 
34. Yun H, Chen L, Xie F, et al. Do patients with moderate or high disease activity escalate rheumatoid arthritis therapy according to treat-to-target principles? Results from the rheumatology informatics system for effectiveness registry of the American College of Rheumatology. Arthritis Care Res. 2020;72(2):166-175. doi:10.1002/acr.24083

35. Solomon DH, Bitton A, Katz JN, Radner H, Brown EM, Fraenkel L. Review: treat to target in rheumatoid arthritis: fact, fiction, or hypothesis? Arthritis Rheumatol. 2014;66(4):775-782. doi:10.1002/art.38323

36. Fleischmann RM, van der Heijde D, Gardiner PV, Szumski A, Marshall L, Bananis E. DAS28-CRP and DAS28-ESR cut-offs for high disease activity in rheumatoid arthritis are not interchangeable. RMD Open. 2017;3(1):e00382. doi:10.1136/rmdopen-2016-000382

37. Smolen JS, Gladman D, McNeil HP, et al. Predicting adherence to therapy in rheumatoid arthritis, psoriatic arthritis or ankylosing spondylitis: a large cross-sectional study. RMD Open. 2019;5(1):e000585. doi:10.1136/rmdopen-2017-000585

38. Murage MJ, Tongbram V, Feldman SR, et al. Medication adherence and persistence in patients with rheumatoid arthritis, psoriasis, and psoriatic arthritis: a systematic literature review. Patient Prefer Adherence. 2018;12:1483-1503. doi:10.2147/PPA.S167508
39. Taylor PC, Alten R, Gomez-Reino JJ, et al. Clinical characteristics and patient-reported outcomes in patients with inadequately controlled rheumatoid arthritis despite ongoing treatment. RMD Open. 2018;4(1): e000615. doi:10.1136/rmdopen-2017-000615

40. van Vilsteren M, Boot CR, Knol DL, et al. Productivity at work and quality of life in patients with rheumatoid arthritis. BMC Musculoskelet Disord. 2015;16:107. doi:10.1186/s12891-015-0562-x

41. Xavier RM, Zerbini CAF, Pollak DF, et al. Burden of rheumatoid arthritis on patients' work productivity and quality of life. $A d v$ Rheumatol. 2019;59(1):47. doi:10.1186/s42358-019-0090-8

42. Fraenkel L, Bogardus S, Concato J, Felson D. Unwillingness of rheumatoid arthritis patients to risk adverse effects. Rheumatology. 2002;41(3):253-261. doi:10.1093/rheumatology/41.3.253

43. Kim H, Xie B. Health literacy in the eHealth era: A systematic review of the literature. Patient Educ Couns. 2017;100 (6):1073-1082. doi:10.1016/j.pec.2017.01.015

44. van der Vaart R, Drossaert CH, de Heus M, Taal E. van de Laar MA. Measuring actual eHealth literacy among patients with rheumatic diseases: a qualitative analysis of problems encountered using Health 1.0 and Health 2.0 applications. J Med Internet Res. 2013;15(2):e27.
Patient Preference and Adherence

\section{Publish your work in this journal}

Patient Preference and Adherence is an international, peer-reviewed, open access journal that focusing on the growing importance of patient preference and adherence throughout the therapeutic continuum. Patient satisfaction, acceptability, quality of life, compliance, persistence and their role in developing new therapeutic modalities and compounds to optimize clinical outcomes for existing disease states are major areas of interest for the journal. This journal has been accepted for indexing on PubMed Central. The manuscript management system is completely online and includes a very quick and fair peer-review system, which is all easy to use. Visit http:// www.dovepress.com/testimonials.php to read real quotes from published authors. 